\title{
Measurements of radio pulsar braking indices
}

\author{
A. E. Chukwude ${ }^{1}$, A. A. Baiden ${ }^{1}$, and C. C. Onuchukwu ${ }^{2}$ \\ 1 Department of Physics \& Astronomy, University of Nigeria, Nsukka, Nigeria \\ e-mail: aus_chukwude@yahoo.com \\ 2 Anambra State University, Uli, Anambra State, Nigeria
}

Received 9 January 2009 / Accepted 5 February 2010

\section{ABSTRACT}

\begin{abstract}
A quantitative analysis of radio pulsar timing noise is presented. Our analysis employs the recently reported strong correlation (with correlation coefficient $r=0.95$ ) between the observed second time derivative of the pulse rotation frequency ( $\ddot{v}_{\text {obs }}$ ), obtained from fully phase-coherent timing analyses, and a timing noise statistic $\left(\sigma_{\mathrm{R} 23}\right)$, used to quantify the amount of pulsar rotational fluctuations absorbed by the coefficient of the cubic term, to isolate the presumed deterministic braking index from the effects attributable to pulsar timing activity. Application of this method to a sample of 27 radio pulsars, whose timing data span 9-13 years, reveals that (i) for 22 pulsars, a sensible braking index measurement was impossible: with numerical values of the braking index either appreciably less than the corresponding uncertainty or anomalously large; (ii) save for the relatively large uncertainties, the braking index appears to be significantly measured in five pulsars. Current results are discussed in the context of the prevailing standard model for radio pulsar spin-down.
\end{abstract}

Key words. methods: statistical - pulsars: general

\section{Introduction}

Accurate measurements of the braking index $n$, which describes how the pulsar spin-down rate varies with its rotation frequency, are fundamental to a better understanding of the pulsar electrodynamics (Yue et al. 2007, and references therein). Currently, the prevailing picture is that pulsars are rapidly rotating, highly magnetised neutron stars dominantly powered by the rotational kinetic energy of the underlying neutron stars (Goldreich \& Julian 1969; Manchester \& Taylor 1977; Shapiro \& Teukolsky 1983). In the context of the widely used standard model, the dominant energy loss mechanism is the pure magnetic dipole radiation at the pulsar rotation frequency and acceleration of particle winds (Pacini 1967; Manchester \& Taylor 1977). The model posits that the spin-down of a pulsar should follow a simple power relation of the form (e.g. Manchester \& Taylor 1977)

$\dot{v}=-K v^{n}$

where $v$ and $\dot{v}$ are, respectively, the pulse rotation frequency and its first time derivative, $K$ is an arbitrary positive constant and $n=3$ is the torque braking index. Equation (1) can be differentiated to obtain an expression for $n$ in terms of the pulsar rotational parameters

$n=\frac{v \ddot{v}}{\dot{v}^{2}}$,

where $\ddot{v}$ is the second derivative of the pulsar spin frequency with respect to time. Equation (2) suggests that measurements of the pulsar braking index could in principle follow directly from the standard pulsar timing technique involving a third-order polynomial model.

However, accurate measurements of $n$ have proven extraordinarily difficult. To date, significant measurements have been reported in about six out of $\sim 1800$ known pulsars. All the six measurements were obtained from the phase-coherent timing analysis (hereafter referred to as PCTA), a technique that relies on accurately accounting for every turn of the pulsar (Lyne, Pritchard \& Smith 1988; Kaspi, et al. 1994; Lyne et al. 1996; Camilo et al. 2000; Livingstone et al. 2006, 2007). The apparent difficulty in measuring a sensible pulsar braking index has been largely attributed to the effects of the pulsar rotational irregularity, most pulsars exhibit a wide range of departures from the assumed spin-down law (Lyne \& Graham-Smith 1998; Lorimer \& Kramer 2005). Broadly speaking, pulsar timing activities can take the form of glitches, spectacular sudden jumps in $v$ and $\dot{v}$, (e.g. Zou et al. 2008, and references therein) and the more generic timing noise, the broad signature of which is observed as unmodelled structures in pulsar barycentric times of arrival (BTOAs) after accounting for the deterministic spin-down (e.g. Hobbs, et al. 2006, and references therein). The pulsar timing activity contaminates the deterministic spin parameters, in most cases, precluding accurate measurements of the relevant spindown parameters. Perhaps, $\ddot{v}$ appears to be most vulnerable to timing activity effects owing to its extremely small amplitude (Chukwude 2007, and references therein).

Recently, Chukwude (2003) carried out a detailed qualitative study of the effects of timing noise on radio pulsar braking index measurements. In particular, the author reported an exceptionally strong correlation between the magnitude of the observed second derivative of the pulse rotation frequency $\left(\ddot{v}_{\text {obs }}\right)$, obtained from the conventional phase-coherent timing analysis and the difference between the root-mean-squares (rms) phase residuals from 2 nd-and 3 rd-order polynomial models $\left(\sigma_{\mathrm{R} 23}\right)$. This result implies that the coefficient of the cubic term, obtained from the standard timing technique, is reasonably contaminated by effects attributable to pulsar timing irregularity. Apparently, effective measurements of the pulsar braking index would hinge on how successfully the deterministic spin-down effect could be decoupled from the often dominant influence of timing activity. Previous efforts to obtain sensible braking index 
measurements have relied largely on various techniques to disentangle timing noise fluctuation effects from the pure pulsar magnetodipole braking. These have taken the form of pre-whitening of the pulse arrival time data (Manchester et al. 1985; Kaspi et al. 1994; Hobbs et al. 2004; Livingstone et al. 2007), partial phase-coherent timing analysis (Lyne et al. 1993) or an outright negation of the second derivative of the pulse rotation frequency (Johnston \& Galloway 1999). Apparently, these techniques have been largely unsuccessful, yielding seemingly realistic braking indices in only six pulsars (Livingstone et al. 2007). A better insight into the true relationship between the radio pulsar timing noise and the braking index has become an indispensible part of the current quest for improved measurements of $n$.

We show that the $\ddot{v}_{\text {obs }}-\sigma_{\mathrm{R} 23}$ relation can equally be employed in quantitative analyses of radio pulsar timing noise. In particular, our analyses apparently yield reasonable estimates for the braking index of some radio pulsars.

\section{Theory of relationships}

Following Chukwude (2003), the frequency second time derivative obtained from a fully phase-coherent timing solution ( $\left.\ddot{v}_{\text {obs }}\right)$ can be modeled in terms of the timing noise and the systematic spin-down components as

$\overline{\ddot{v}}_{\text {obs }}=\ddot{v}_{\text {tno }}+\ddot{v}_{\text {dip }}$,

where $\ddot{v}_{\text {tno }}$ is the component that originates from all forms of fluctuations in the pulsar clock (unresolved glitches, microglitches and timing noise), $\ddot{v}_{\text {dip }}$ is the deterministic spin-down component (presumably from pure magnetodipole radiation) and $\overline{\vec{v}}_{\text {obs }} \equiv\left|\ddot{v}_{\text {obs }}\right|$ is the magnitude of the observed braking index. This model makes at least two elegant predictions. Firstly, the coefficient of the cubic term merely models the deterministic pulsar spin-down by way of pure magnetic dipole torque braking (Manchester \& Taylor 1977). This requires that $\ddot{v}_{\text {tno }} \ll \ddot{v}_{\text {dip }}$ and $\ddot{v}_{\text {obs }} \simeq \ddot{v}_{\text {dip }}$. In this context, $\ddot{v}_{\text {obs }}$ is expected to yield $n_{\text {dip }} \simeq 3$. This is most probably the case for the five young pulsars whose braking indices have been significantly measured via PCTA technique (e.g. Livingstone et al. 2007).

An alternative scenario is that $\ddot{v}_{\text {obs }}$ predominantly quantifies the level of the pulsar rotational irregularity. The latter scenario will require $\ddot{v}_{\text {tno }} \gg \ddot{v}_{\text {dip }}$ and $\ddot{v}_{\text {obs }} \simeq \ddot{v}_{\text {tno }}$ (but $\ddot{v}_{\text {dip }}$ is not necessarily equal to zero). These conditions will result in non-stationary braking indices, with either positive or negative values. In particular, the braking indices obtained from $\ddot{v}_{\text {obs }}$ could have anomalous values, some several orders of magnitude greater than or less than the canonical value of 3 (Johnston \& Galloway 1999; Chukwude 2003; Hobbs et al. 2004). Moreover, $\ddot{v}_{\text {obs }}$ will almost certainly correlate with some timing noise statistics. A statistic of interest is the difference between the root-mean-squares phase residuals obtained from 2 nd- and 3rd-order polynomial models $\left(\sigma_{\mathrm{R} 23}\right)$. Using a sample of 27 radio pulsars, Chukwude (2003) shows that $\sigma_{\mathrm{R} 23}$ is about $95 \%$ correlated with timing noise dominated $\left|\ddot{v}_{\text {obs }}\right|$. This is perhaps the most plausible scenario for the majority of the known radio pulsars. Following Chukwude (2003), we redefine the timing noise statistic $\left(\sigma_{\mathrm{R} 23}\right.$ : a measure of timing noise activity absorbed by the observed $\ddot{v})$ as

$\sigma_{\mathrm{R} 23}=\sqrt{\sigma_{\mathrm{R} 2}^{2}(2, T)-\sigma_{\mathrm{R} 3}^{2}(3, T)}$,

where $\sigma_{\mathrm{R} 2}(2, T)$ and $\sigma_{\mathrm{R} 3}(3, T)$ are the root-mean-square (rms) phase residuals from 2 nd- and 3rd-order polynomial fits to the BTOA data, respectively and $T$ is the data timespan length. On the premise that the latter scenario is the prevailing case for most known radio pulsars (especially those with $\tau_{c} \geq 5 \mathrm{kyr}$ ), we posit a simple power-law relation between the observed braking index and the timing irregularity statistic of the form

$\ddot{v}_{\mathrm{tno}}=A \sigma_{\mathrm{R} 23}^{\beta}$,

where we take that $A$ and $\beta$ (power law index) are constants for a given sample. Theoretically, $A$ represents the smallest value of $\ddot{v}$ for the sample, with minimal timing activity contamination. We used the condition that for the current scenario $\overline{\vec{v}}_{\text {obs }} \simeq \ddot{v}_{\text {tno }}$ to arrive at Eq. (5). Once $A$ and $\beta$ are obtained for a sample of radio pulsars, Eq. (5) can be used to estimate the timing activity component of the pulsar braking index $\left(\ddot{v}_{\text {tno }}\right)$. The deterministic component of the frequency second derivative is given by

$\ddot{v}_{\text {dip }}=\left|\overline{\vec{v}}_{\text {obs }}-\ddot{v}_{\text {tno }}\right|$.

The choice of definition of $\ddot{v}_{\text {dip }}$ in Eq. (6) is necessitated by the non-stationary character of $\ddot{v}_{\text {obss }}$.

\section{Observations and data analyses}

Regular timing observations of all pulsars in the current sample commenced at Hartebeesthoek Radio Astronomy Observatory between 1984 January and 1987 May and are still ongoing. However, a major interruption in the HartRAO pulsar timing program occurred between 1999 June and 2000 August during a major hardware upgrade. Save for pulsars B0833-45 and B1641-45, which were on a real time glitch monitoring program, no pulsar was observed during this period. Observations were made regularly at intervals of $\lesssim 14$ days near either 1668 or $2272 \mathrm{MHz}$ with the 26-m HartRAO radio telescope. Pulses were recorded with a single $10 \mathrm{MHz}$ bandwidth receiver at both frequencies and no pre-detection dedispersion hardware was implemented during the period. Detected pulses were smoothed with an appropriate filter-time constant, and integrated over $N_{\mathrm{p}}$ consecutive rotation periods, where $N_{\mathrm{p}}$ is different for different pulsars. For the present sample of 27 radio pulsars, the values of $N_{\mathrm{p}}$ lie between 500 and 5000 , corresponding to integration times ranging between $\sim 48 \mathrm{~s}$ and $32 \mathrm{~min}$. An integration was usually started at a particular second by synchronization to the station clock, which was derived from a hydrogen maser and was referenced to the Universal Coordinated Time (UTC) via a Global Positioning Satellite (GPS) network.

All topocentric arrival times obtained at HartRAO between 1984 and 1999 were transformed to infinite observing frequency at the Solar System Barycentre (SSB) with the Jet Propulsion Laboratory DE200 solar system ephemeris and the TEMPO software package (http: //pulsar.princeton. edu/tempo). Subsequent modelling of the resulting barycentric times of arrival (BTOAs) was accomplished with the HartRAO in-house timing analysis software, which is based on the standard pulsar timing technique of Manchester \& Taylor (1977) and is well described in Flanagan (1995). At the solar system barycentre, the time evolution of the rotational phase of a non-binary pulsar is better studied by fitting the BTOAs with a Taylor series expansion of phase of the form (e.g. Manchester \& Taylor 1977)

$\phi(t)=\phi_{0}+v\left(t-t_{0}\right)+\frac{1}{2} \dot{v}\left(t-t_{0}\right)^{2}+\frac{1}{6} \ddot{v}\left(t-t_{0}\right)^{3}$,

where $\phi_{0}$ is the phase at an arbitrary time $t_{0}$. In practice, for sufficiently accurate values of $v, \dot{v}$ and $\ddot{v}$, Eq. (7) was used to predict the phase of a given pulsar at any time $t$. Usually, the 
Table 1. Results of the observed and calculated parameters of the 27 HartRAO Pulsars.

\begin{tabular}{|c|c|c|c|c|c|c|c|c|c|c|c|c|c|}
\hline $\begin{array}{l}\text { Object } \\
\text { (PSR B) } \\
(1)\end{array}$ & $\begin{array}{c}v \\
\left(\mathrm{~s}^{-1}\right) \\
(2) \\
\end{array}$ & $\begin{array}{c}\Delta v^{a} \\
\left(\mathrm{~s}^{-1}\right) \\
(3)\end{array}$ & $\begin{array}{l}\dot{v} \\
(\S) \\
(4)\end{array}$ & $\begin{array}{r}\Delta \dot{v}^{a} \\
(\S) \\
(5)\end{array}$ & $\begin{array}{l}\ddot{v}_{\text {obs }} \\
(\ddagger) \\
(6)\end{array}$ & $\begin{array}{c}\Delta \ddot{v}_{\text {obs }}{ }^{a} \\
(\ddagger) \\
(7)\end{array}$ & $\begin{array}{c}\sigma_{\mathrm{R} 23} \\
(\mathrm{mP}) \\
(8)\end{array}$ & $\begin{array}{l}\ddot{v}_{\text {tno }} \\
(\ddagger) \\
(9)\end{array}$ & $\begin{array}{c}\Delta \ddot{v}_{\text {tno }} \\
(\ddagger) \\
(10)\end{array}$ & $\begin{array}{c}\ddot{v}_{\mathrm{dip}} \\
(\ddagger) \\
(11)\end{array}$ & $\begin{array}{c}\Delta \ddot{\nu}_{\text {dip }} \\
(\ddagger)\end{array}$ & $n_{\text {dip }}$ & $\Delta n_{\text {dip }}$ \\
\hline $0450-18$ & 1.821696158392 & 6 & -19.091821 & 18 & 0.051 & 12 & 1.3 & 0.067 & 0.21 & 0.016 & 0.210 & 8 & 105 \\
\hline $0736-40$ & 2.66723899983 & 7 & -11.467300 & 16 & -7.674 & 6 & 280.6 & 15.26 & 0.46 & 7.59 & 0.46 & 15386 & 900 \\
\hline 0740-28 & 5.99657769025 & 6 & -604.73385 & 6 & -35.54 & 8 & 620.9 & 34.04 & 0.80 & 1.50 & 0.80 & 2.5 & 1.3 \\
\hline $0835-41$ & 1.330454723903 & 9 & -6.271580 & 5 & 0.3795 & 10 & 8.5 & 0.444 & 0.026 & 0.0647 & 0.0260 & 219 & 88 \\
\hline 0959-54 & 0.69609220478 & 6 & -24.994522 & 12 & -13.827 & 4 & 260.3 & 14.15 & 0.42 & 0.323 & 0.420 & 35 & 39 \\
\hline $1054-62$ & 2.36715607565 & 7 & -20.01190 & 4 & -0.326 & 6 & 4.4 & 0.23 & 0.16 & 0.094 & 0.160 & 56 & 95 \\
\hline $1133+16$ & 0.84181190896 & 12 & -2.645946 & 6 & 0.0652 & 8 & 2.2 & 0.114 & 0.016 & 0.049 & 0.017 & 593 & 196 \\
\hline $1221-63$ & 4.619428499992 & 10 & -105.71397 & 4 & -0.1410 & 10 & 4.0 & 0.21 & 0.12 & 0.069 & 0.120 & 2.9 & 4.9 \\
\hline $1240-64$ & 2.574123963978 & 5 & -29.819964 & 12 & -0.744 & 4 & 14.3 & 0.647 & 0.057 & 0.097 & 0.058 & 28 & 17 \\
\hline $1323-58$ & 2.09208875280 & 6 & -14.12989 & 6 & 4.402 & 6 & 39.1 & 2.082 & 0.090 & 2.320 & 0.090 & 2430 & 90 \\
\hline $1323-62$ & 1.88709280930 & 5 & -67.258115 & 14 & -1.706 & 4 & 33.1 & 1.764 & 0.060 & 0.058 & 0.060 & 2.4 & 2.5 \\
\hline $1356-60$ & 7.84290777286 & 4 & -389.86801 & 7 & 5.06 & 7 & 85.2 & 4.58 & 0.14 & 0.48 & 0.15 & 2.5 & 0.8 \\
\hline $1358-63$ & 1.186530997525 & 6 & -23.58777 & 5 & 8.692 & 8 & 160.2 & 8.66 & 0.23 & 0.027 & 0.23 & 6 & 50 \\
\hline $1426-66$ & 1.273169693773 & 7 & -4.489741 & 12 & -0.190 & 9 & 2.4 & 0.126 & 0.098 & 0.064 & 0.099 & 400 & 630 \\
\hline $1449-64$ & 5.57148949973 & 5 & -85.23778 & 6 & 1.592 & 10 & 29.2 & 1.55 & 0.16 & 0.041 & 0.160 & 3 & 10 \\
\hline $1451-68$ & 3.796841136660 & 12 & -1.424633 & 10 & -0.037 & 4 & 0.5 & 0.023 & 0.177 & 0.014 & 0.178 & 2619 & 33300 \\
\hline $1556-44$ & 3.890198835898 & 7 & -15.42827 & 5 & 0.212 & 8 & 2.6 & 0.137 & 0.088 & 0.075 & 0.088 & 123 & 144 \\
\hline $1557-50$ & 5.192073158034 & 6 & -136.46835 & 6 & -0.046 & 7 & 2.8 & 0.145 & 0.030 & 0.099 & 0.030 & 2.8 & 0.8 \\
\hline $1641-45$ & 2.197513500235 & 11 & -97.11850 & 8 & 4.1687 & 6 & 85.7 & 4.60 & 0.14 & 3.43 & 0.14 & 80 & 3 \\
\hline $1642-03$ & 2.57938001879 & 6 & -11.85138 & 5 & -0.123 & 6 & 8.2 & 0.428 & 0.065 & 0.305 & 0.065 & 560 & 120 \\
\hline $1706-16$ & 1.531264844912 & 8 & -14.73178 & 4 & 6.341 & 7 & 85.8 & 4.61 & 0.16 & 1.73 & 0.16 & 1222 & 110 \\
\hline $1727-47$ & 1.205132491642 & 18 & -237.60720 & 5 & 3.560 & 8 & 43.5 & 2.278 & 0.068 & 1.282 & 0.050 & 2.7 & 0.2 \\
\hline $1749-28$ & 1.777590485696 & 20 & -25.670282 & 9 & -0.099 & 4 & 10.7 & 0.564 & 0.048 & 0.465 & 0.048 & 125 & 13 \\
\hline 1822-09 & 1.30042174971 & 8 & -88.36158 & 10 & 14.262 & 6 & 258.6 & 14.05 & 0.36 & 0.21 & 0.36 & 3.5 & 5.9 \\
\hline $1929+10$ & 4.41466176139 & 15 & -22.542298 & 17 & -2.185 & 7 & 12.5 & 0.657 & 0.085 & 1.528 & 0.085 & 1327 & 75 \\
\hline $1933+16$ & 2.78753800616 & 14 & -46.638826 & 14 & 0.145 & 10 & 3.3 & 0.172 & 0.065 & 0.027 & 0.066 & 3.4 & 8.3 \\
\hline 2045-16 & 0.509794578169 & 12 & -2.848134 & 7 & 0.0086 & 12 & 0.1 & 0.0052 & 0.0350 & 0.0034 & 0.0350 & 21 & 216 \\
\hline
\end{tabular}

Notes. ${ }^{(a)}$ Errors are 2- $\sigma$ formal standard errors and refer to the last significant digit. $\S$ In units of $10^{-15} \mathrm{~s}^{-2}$. In units of $10^{-25} \mathrm{~s}^{-3}$.

BTOAs and initial pulsar rotation parameters constitute the input to the timing analysis software. The output consists of the refined spin-down parameters and timing residuals (the difference between the observed and model-predicted arrival times). Following Cordes \& Downs (1985), the phase residuals, $\mathfrak{R}\left(t_{\mathrm{j}}\right)$ for $1 \leq j \leq N$, where $N$ is the number of observations, are used to calculate the root-mean-square phase residuals $\sigma_{\mathrm{R}}(m, T)$ (where $m=2$ and 3 for 2nd- and 3rd-order polynomial fits, respectively, and $T$ is the observation time span).

\section{Results}

The relevant measured and derived parameters of the 27 HartRAO pulsars are summarized in Table 1. Column 1 contains the pulsar name using the B1950.0 naming convention; Cols. 2 and 3 list the spin frequency and the associated formal standard error; the spin-down rate and its formal error are contained in Cols. 4 and 5; Cols. 6-8 list, respectively, the observed frequency second derivative, its formal standard error and the timing activity statistic; the calculated timing activity component of $\ddot{v}$ and its formal error are listed in Cols. 9 and 10, respectively; Cols. 11 and 12 contain the presumed deterministic component of $\ddot{v}$ and the associated formal error, respectively, while the resulting braking index $\left(n_{\text {dip }} \equiv \ddot{v}_{\text {dip }} v / \dot{v}^{2}\right)$ and the formal standard error are listed in Cols. 13 and 14, respectively. The quoted uncertainties are 2- $\sigma$ formal standard errors and refer to the least significant figures in Cols. 2, 4, 6 and 9 only. The uncertainties in $v, \dot{v}$ and $\ddot{v}_{\text {obs }}$ were obtained directly from HartRAO in-house timing analysis software and were calculated for other parameters. The quoted uncertainties in $\ddot{v}_{\text {tno }}$ and $n_{\text {dip }}\left(\Delta \ddot{v}_{\text {tno }}\right.$ and $\Delta n_{\text {dip }}$, respectively) are basically estimates of the standard errors in products and/or quotients of uncorrelated variables. Specifically, $\Delta \ddot{v}_{\text {tno }}=\ddot{v}_{\text {tno }}\left\{(\Delta A / A)^{2}+(\Delta \beta / \beta)^{2}+\left(\sigma_{\mathrm{W}} / \sigma_{\mathrm{R} 23}\right)^{2}\right\}^{1 / 2}:$ where, following Eq. (5), $\Delta A$ and $\Delta \beta$ are respectively the standard errors in the intercept and slope of the graph in Fig. 1a and $\sigma_{\mathrm{W}}$ is the rms white noise of the observed phase residuals. Similarly, $\Delta n_{\text {dip }}=n_{\text {dip }}\left\{(\Delta v / v)^{2}+\left(\Delta \ddot{v}_{\text {dip }} / \ddot{v}_{\text {dip }}\right)^{2}+(2 \Delta \dot{v} / \dot{v})^{2}\right\}^{1 / 2}$, where $\Delta v$ and $\Delta \dot{v}$ are the uncertainties in the pulsar rotation frequency and its first time derivative, respectively. The uncertainty in $\ddot{v}_{\text {dip }}\left(\Delta \ddot{v}_{\text {dip }}\right)$ is the quadrature sum of the standard errors in and $\ddot{v}_{\text {obs }}$ and $\ddot{v}_{\text {tno. }}$.

The uncertainties in $\sigma_{\mathrm{R} 23}$ were estimated from the real scatter in the phase residuals. The scatter in the BTOAs arises mainly from pulse phase jitter and measurement uncertainty and will be characteristic of white noise (Cordes \& Downs 1985). The resultant rms white noise in the observed phase residuals $\left(\sigma_{\mathrm{W}}\right)$ was estimated with pairs of data from the 2 nd-order timing models, separated by the time interval $\Delta t \leq 1$ day. The short time scale is required to filter out the more slowly varying red noise component from the white noise estimator (Cordes \& Downs 1985; Chukwude 2002). As expected, estimates of $\sigma_{\mathrm{W}}$ represent the upper limit on the error in $\sigma_{\mathrm{R} 23}$. We quantify the level of rotational fluctuations in our current sample of pulsars with the 

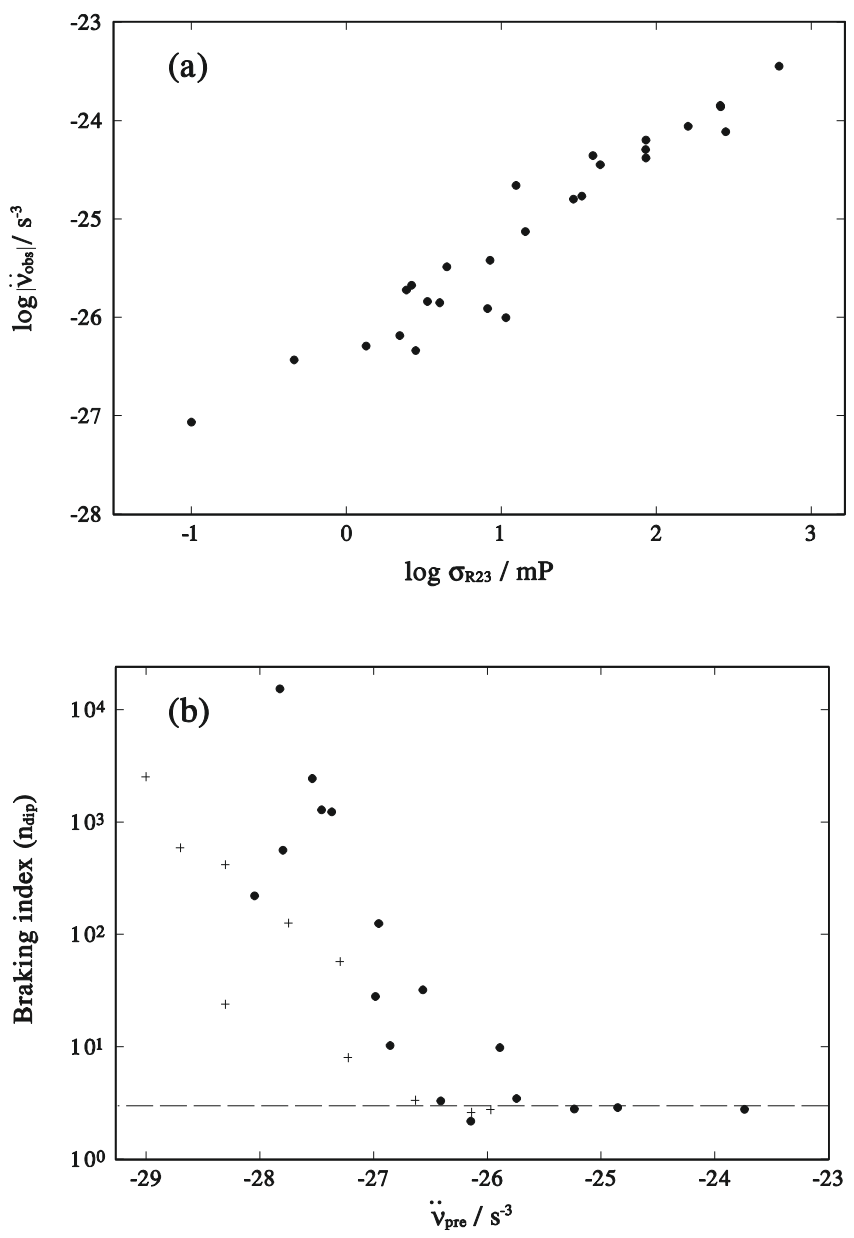

Fig. 1. Log- log scatter plots of (a) the absolute magnitude of the observed frequency second time derivative $\left(\left|\ddot{v}_{\text {obs }}\right|\right)$ against the timing noise activity paramater $\left(\sigma_{\mathrm{R} 23}\right)$, obtained as described in the text, and (b) the calculated braking indices $\left(n_{\text {dip }}\right)$, against $\ddot{v}_{\text {pre }} \equiv 3 \dot{v}^{2} / v$ (the expected deterministic frequency second derivative, assuming a standard vacuum magnetodipole radiation model with $n=3$. The long dashed horizontal line indicates $n_{\text {dip }}=3$. Key: $\bullet=$ pulsars exhibiting pronounced timing activity $\left(\sigma_{\mathrm{R} 23}>5 \sigma_{\mathrm{W}} ;+=\right.$ pulsars with notable low timing activity level $\left(\sigma_{\mathrm{R} 23}<5 \sigma_{\mathrm{W}}\right)$.

ratio $\sigma_{\mathrm{R} 23} / \sigma_{\mathrm{W}}$ (hereafter referred to as the timing activity-tonoise ratio, TNR). Pulsars whose phase residuals display large amplitude intrinsic scatter are generally characterised by high values of $\sigma_{\mathrm{W}}$, corresponding to low TNR.

Figure 1 shows on $\log -\log$ scales the plots of the absolute values of the observed frequency second derivative $\left(\left|\ddot{v}_{\text {obs }}\right|\right)$ against the timing noise statistic, $\sigma_{\mathrm{R} 23}$ (Fig. 1a) and the measured braking indices $\left(n_{\text {dip }}\right)$ against the presumed deterministic frequency second derivative, $\ddot{v}_{\text {pre }}$ (Fig. 1b). Figure 1a shows that the current definition of the pulsar timing activity statistic minimised the scatter in the $\ddot{v}_{\mathrm{obs}}-\sigma_{\mathrm{R} 23}$ plot (see Chukwude 2003, Fig. 1a). A simple linear regression analysis of the data in Fig. 1a yields a correlation coefficient $r=+0.97$, which is a slight improvement over $\sim+0.95$ reported for the two variables by Chukwude (2003). The marginal increase could be caused by the current definition, which yields $\sigma_{\mathrm{R} 23}$ values that are systematically higher than those reported in Chukwude (2003) and reduces the dispersion in the values by about a factor of 6 . In particular, we find the exact form of relationship between the two variables as

$\log \left|\ddot{v}_{\text {obs }}\right|=-26.29 \pm 0.05+(1.01 \pm 0.03) \log \sigma_{\mathrm{R} 23}$.

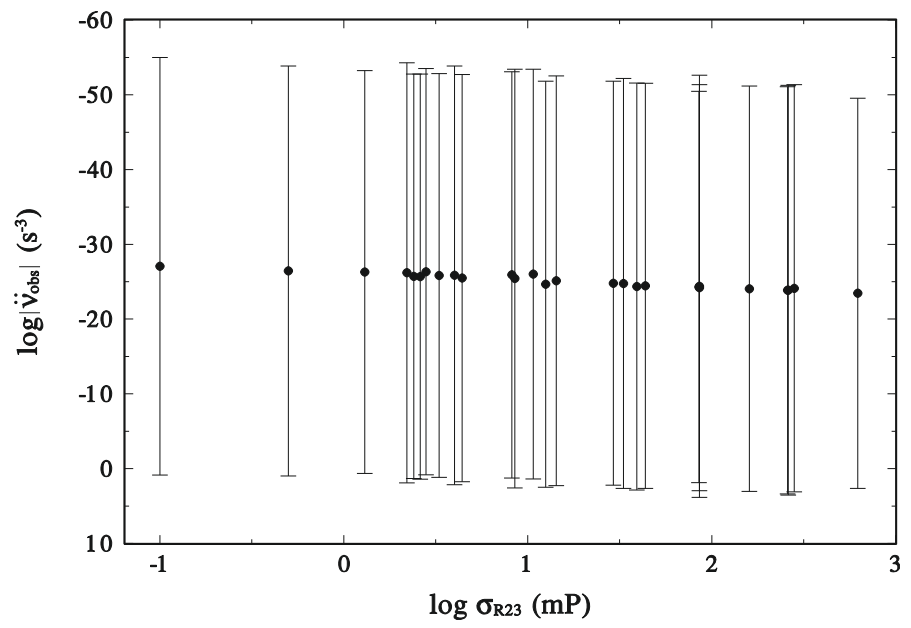

Fig. 2. $\log -\log$ plot of $\left|\ddot{v}_{\text {obs }}\right|$ against $\sigma_{\mathrm{R} 23}$ with the quoted uncertainties in $\ddot{v}_{\text {obs }}$ included. Error bars are $2-\sigma$ formal standard errors.

The quoted errors are 2- $\sigma$ formal standard errors and refer to the least significant digits. The strength of the correlation reaffirms earlier results (Chukwude 2003; Hobbs et al. 2004) that the braking indices of most pulsars measured from PCTA are severely contaminated by effects attributable to pulsar timing activity (i.e. $\left|\ddot{v}_{\text {obs }}\right| \simeq \ddot{v}_{\text {tno }}$ ). Estimates of $\ddot{v}_{\text {tno }}$ and $\ddot{v}_{\text {dip }}$ follow directly from Eqs. (5) and (6), respectively. It is apparent from Fig. 1b that the current method resulted in $n_{\text {dip }} \sim 3$ for most pulsars with $\ddot{v}_{\text {pre }} \gtrsim 5 \times 10^{-27} \mathrm{~s}^{-3}$, irrespective of the size of the error bars. The departure from the canonical value of 3 , however, increases sharply below $5 \times 10^{-27} \mathrm{~s}^{-3}$. For a given value of $\ddot{v}_{\text {pre }}<5 \times 10^{-27} \mathrm{~s}^{-3}$, the amplitude of the departure appears smaller for pulsars with notable weak timing activity $\left(\sigma_{\mathrm{R} 23} / \sigma_{\mathrm{W}}<5\right)$. Figure 2 allows us to appreciate the magnitude of uncertainties in the observed braking indices $\left(\ddot{v}_{\text {obs }}\right)$. In particular, it shows that the size of the errors in the parameter is so large that it completely buries the strong trend in the $\left|\ddot{v}_{\text {obs }}\right|-\sigma_{\mathrm{R} 23}$ data (see Fig. 1a).

\section{Discussion}

The spread in both the spin-down rates $(\dot{v})$ and characteristic ages $\left(\tau_{c}\right)$ of the pulsars in the HartRAO sample covers $\sim 2$ orders of magnitude $\left(\sim 1.0 \times 10^{-15}<|\dot{v}|<610 \times 10^{-15} \mathrm{~s}^{-2}\right.$ and $70<\tau_{c}<43000 \mathrm{kyr}$, respectively). Aside from two pulsars (B1451-68 and B2045-16, with $\tau_{c}>10000 \mathrm{kyr}$ ) the remainder of 25 pulsars have $\tau_{c}<5$ Myr and can loosely be classified as middle-aged pulsars. Pulsars in this class are widely believed to support a range of enhanced rotational instabilities, particularly timing noise and microglitches (e.g. Chukwude \& Urama 2010; Chukwude 2007; D’Alessandro et al. 1995; Cordes \& Downs 1985). The results of the quantitative analyses of the $\ddot{v}_{\mathrm{obs}}-\sigma_{\mathrm{R} 23}$ relation for current sample show that

(i) for about 22 pulsars, the numerical values of the measured braking index are either significantly less than the corresponding standard formal uncertainties or are anomalously large $\left(n_{\text {dip }} \gg 3\right)$;

(ii) for the remainder of five pulsars with relatively high spindown rates $\left(|\dot{v}|>50 \times 10^{-15} \mathrm{~s}^{-2}\right)$, we obtain apparently sensible values $\left(n_{\text {dip }} \sim 3\right)$ for the braking index.

There are two major sources of uncertainties in the calculated braking indices. Firstly, small number statistics limit the 
measurement precision to $\gtrsim 2.5 \%$ of the parameter values for objects in the current sample. A more serious limitation is placed on the accuracy of our results by the numerical values of TNR. Uncertainties in $\sigma_{\mathrm{R} 23}$, the rms white noise component of the observed phase residuals, could introduce errors ranging between $\sim 0.2$ and $700 \%$ of the numerical value of $n_{\text {dip. }}$. Apparently, the quoted formal errors in the calculated parameters of pulsars with very low TNR $\left(\sigma_{\mathrm{R} 23} / \sigma_{\mathrm{W}} \lesssim 10\right)$ are dominated by the uncertainty in $\sigma_{\mathrm{R} 23}$. Quantitatively, the uncertainties in $\ddot{v}_{\text {tno }}$ for the highest and lowest TNR pulsars in the sample amount to $\sim 2.7 \%$ and $700 \%$ respectively of the parameter values. This error basically propagates through all subsequently calculated parameters $\left(\Delta \ddot{v}_{\text {dip }}^{2}=\Delta \ddot{v}_{\text {obs }}^{2}+\Delta \ddot{v}_{\text {tno }}^{2}\right)$. As a result, the uncertainty in $\ddot{v}_{\text {dip }}$ consists of $\sim 2.7-700 \%$ of $\ddot{v}_{\text {tno }}$, which could be several factors of 10 higher than the numerical value of $\ddot{v}_{\text {dip }}$ (since $\Delta \ddot{v}_{\text {obs }} \ll \Delta \ddot{v}_{\text {tno }}$ ). The formal error in $n_{\text {dip }}$ scales as $\sim \Delta \ddot{v}_{\text {dip }} / \ddot{v}_{\text {dip }}$ and would be anomalously large for pulsars having $\Delta \ddot{v}_{\text {dip }} \gg \ddot{v}_{\text {dip }}$.

About nine pulsars show apparently a very low level of timing noise activity $\left(\sigma_{\mathrm{R} 23} / \sigma_{\mathrm{W}}<5\right)$. For these pulsars, $n_{\text {dip }}$ is $\sim 3$ and $>8$ for three and six objects, respectively. The estimated uncertainties in the braking indices are unusually large, in most cases exceeding the numerical value of the observed $n_{\text {dip }}$. We surmise that the observed low TNR might not be an indication of improved rotational stability of the affected pulsars. We identify two major factors that could give rise to low $\sigma_{\mathrm{R} 23} / \sigma_{\mathrm{W}}$ in pulsars with intrinsically measurable rotational activity. Firstly, all objects, for which the 3rd-order polynomial fits do not model the data significantly better than the 2nd-order fits are characterised by a low TNR. For these pulsars (e.g. B1054-62 and B1557-50), $\sigma_{\mathrm{R} 2}(2, T) \simeq \sigma_{\mathrm{R} 3}(3, T)$ and $\sigma_{\mathrm{R} 23}$ will be unusually small. These pulsars have been shown to exhibit reasonable level of timing activity, $\sigma_{\mathrm{R} 2}(2, T) / \sigma_{\mathrm{W}}>10$, and may require higher order polynomial terms (Chukwude 2002; D'Alessandro et al. 1995). Specifically, the pulsar B1557-50 has been shown to exhibit dominant periodic timing variations (Chukwude et al. 2003). Secondly, the observed small $\sigma_{\mathrm{R} 23} / \sigma_{\mathrm{W}}$ could be an artifact of the HartRAO local observing system parameters narrow single channel receiver bandwidth, high observing frequencies and short $(\$ 30 \mathrm{~min})$ integration time. These parameters would almost certainly conspire to degrade the quality of the measured pulse arrival times. Consequently, the observed BTOAs residuals are dominated by large $\left(\sigma_{\mathrm{W}}>3 \mathrm{mP}\right)$ intrinsic scatter, which could effectively swamp any form of smaller amplitude timing activity inherent in these pulsars. Remarkably, the timing noise activity of some of these pulsars have been studied in some details elsewhere (e.g. Cordes \& Downs 1985; D’Alessandro et al. 1995).

But for the relatively large uncertainties, the braking index appears to be significantly measured in at least five of the 27 pulsars. These pulsars - B0740-28 (2.5 \pm 1.3$)$, B1323-62 $(2.4 \pm 2.5), \mathrm{B} 1356-60(2.5 \pm 0.8), \mathrm{B} 1557-50(2.8 \pm 0.8)$ and B 1727-47 $(2.7 \pm 0.2)$ - are all moderately spinning down $|\dot{v}| \sim$ $50 \times 10^{-15}-600 \times 10^{-15} \mathrm{~s}^{-2}$. Save for PSR B0740-28 (with $\tau_{c} \sim$ $1500 \mathrm{kyr}$ ), all are younger than 600 kyr. Recently, Chukwude (2007) noted that the spin-down rate of $\sim-605 \times 10^{-15} \mathrm{~s}^{-2}$ associated with the pulsar is atypical for objects of a similar spin-down age. In addition, the five pulsars are characterised by $\ddot{v}_{\text {pre }} \simeq 0.05 \times 10^{-25}-1.51 \times 10^{-25} \mathrm{~s}^{-3}$. Theoretically, braking indices of these sizes could contribute $\sim 60-1800 \mathrm{mP}$ in a pulsar phase over a 13-yr span of data. However, it is unlikely that the effects produced by these phase changes could be measurable in the face of the prevalent and more dominant effects of timing irregularity (Chukwude 2002). Perhaps the most striking of the measurements is the $n_{\text {dip }}=2.7 \pm 0.2$ obtained for the pulsar
B1727-47. Incidentally, some of the parameters of this pulsar $\left(\tau_{c}=78 \mathrm{kyr}\right.$ and $\left.\dot{v} \sim-237 \times 10^{-15} \mathrm{~s}^{-2}\right)$ are comparable, at least at the same order of magnitude, with those of the six pulsars, for which braking indices have been significantly measured (Lorimer \& Kramer 2005). However, the measurement might change significantly for different spans of data.

Sensible values of the pulsar braking index from an analysis of timing activity statistics $\left(\ddot{v}_{\mathrm{obs}}\right.$ and $\left.\sigma_{\mathrm{R} 23}\right)$ has a far reaching implication for the standard pure magnetodipole spin-down model. A first guess is that the result indicates the overall success of the current technique in measuring the braking index of some relatively slow rotating pulsars. Unfortunately, there has been no previous claim of significant braking index measurements from this category of pulsars. This interpretation appears even more doubtful given that both $\ddot{v}_{\mathrm{obs}}$ and $\sigma_{\mathrm{R} 23}$ are widely reputed to be parameters that quantify random fluctuations in the pulse rotation phase (Chukwude 2003; Hobbs et al. 2004) and are expected to be highly variable. Consequently, the resulting braking indices are expected to be nonstationary and very unreliable. In this case, the current apparently sensible values of $n_{\text {dip }}$ might just be incidental and would be extremely difficult to be accepted as real. Nonetheless, the measurements suggest that the much sought-after radio pulsar braking index of $\sim 3$ may not constitute unique measurements.

The current paucity of systematic pulsar braking index measurements can be attributed to deviant rotational behaviours glitches, microglitches and timing noise. Johnston \& Galloway (1999) have shown how pulsar glitch activities within and outside the timing solution could possibly produce spurious braking indices of variable sizes and signatures. Notably three of the current 27 objects have glitched at least once during the period under investigation (Melatos et al. 2008). Thus it is possible that the $\ddot{v}_{\text {obs }}$ reported for most of these pulsars are significantly dominated by glitch effects. Very recently, Chukwude \& Urama (2010) demonstrated that microglitch events are more widespread and occur more frequently in most of the 27 HartRAO pulsars. Accumulations of microglitches of different signs could adequately account for anomalous pulsar braking indices (Chukwude, in prep.). On the other hand, Urama, Link \& Weisberg (2006) have argued that $n \neq 3$ could arise from noisy fluctuations in the external spin-down torque (the so-called timing noise). The authors showed that even marginal fluctuations in the braking torque ( $\sim 1$ part in 200$)$ could yield $\ddot{v}_{\text {obs }}$ of different signs, with magnitudes that are several factors of 10 higher than the canonical value of 3 . Incidentally, timing noise is particularly prevalent among most radio pulsars, including the current objects (Lorimer \& Kramer 2005). Thus it is possible that the $\ddot{v}_{\text {obs }}$ reported for most of current pulsars largely quantifies the effects of deviant rotational behaviour.

\section{Conclusion}

We have conducted a quantitative analysis of timing noise in radio pulsars, using the $\ddot{v}_{\text {obs }}-\sigma_{\mathrm{R} 23}$ relation of a sample of 27 HartRAO pulsars. Our method, which mostly gave braking indices with anomalous values, fails to support the hypothesis that pure magnetic dipole radiations at pulsar rotation frequency and acceleration of particle winds constitute the dominant spin-down mechanism for most radio pulsars. Our data are largely consistent with the prevailing paradigm that the systematic smooth spin-down of most radio pulsars is overshadowed by effects due to rotational instabilities. Nonetheless, we obtained seemingly reasonable measurements $\left(n_{\text {dip }} \sim 3\right)$ for the braking index of five pulsars, which perhaps introduced some 
ambiguities in the interpretation of the a priori assumed radio pulsar braking index of 3 .

Acknowledgements. This work was done in part while AEC was visiting the Abdus Salam International Centre for Theoretical Physics, Trieste, Italy as a Junior Associate. He is grateful to the Swedish International Development Agency (SIDA) for facilitating his visit to ICTP with a travel grant. AEC acknowledges the Director of HartRAO and Dr. C.S. Flanagan for the pulsar data. The authors are grateful to an anonymous referee for very useful comments and suggestions that helped to improve this paper.

\section{References}

Camilo, F., Kaspi, V. M., Lyne, A. G., et al. 2000, ApJ, 541, 367

Chukwude, A. E. 2002, PhD Thesis, University of Nigeria, Nsukka

Chukwude, A. E. 2003, A\&A, 406, 667

Chukwude, A. E. 2007, Chin. J. Astron. Astrophys. 7, 521

Chukwude, A. E., Ubachukwu, A. A., \& Okeke, P. N. 2003, A\&A, 399, 231

Chukwude, A. E., \& Urama, J. O. 2010, MNRAS, submitted

Cordes, J. M., \& Downs, G. S. 1985, ApJS, 59, 343

D’Alessandro, F., McCulloch, P. M., Hamilton, P. A., \& Deshpande, A. A. 1995, MNRAS, 277, 1033

Flanagan, C. S. 1995, PhD Thesis, Rhodes University, Grahamstown, South Africa
Goldreich, P., \& Julian, W. H. 1969, ApJ, 157, 869

Hobbs, G., Lyne, A. G., Kramer, M., et al. 2004, MNRAS, 353, 1311

Hobbs, G., Lyne, A. G., \& Kramer, M. 2006, Chin. J. Astron. Astrophys. 6, 169 Johnston, S., \& Galloway, D. 1999, MNRAS, 306, L50

Kaspi, V. M., Manchester, R. N., Siegman, B., et al. 1994, ApJ, 422, L83

Livingstone, M. A., Kaspi, V. M., Gotthelf, E. V., \& Kuiper, L. 2006, ApJ, 647, 1286

Livingstone, M. A., Kaspi, V. M., Gavriil, F. P., et al. 2007, Ap\&SS, 308, 317

Lorimer, D., \& Kramer, M. 2005, Handbook of Pulsar Astronomy (Cambridge University Press)

Lyne, A. G., \& Graham-Smith, F. 1998, Pulsar Astronomy (Cambridge University Press)

Lyne, A. G., Pritchard, R. S., \& Smith, F. G. 1988, MNRAS, 233, 667

Lyne, A. G., Pritchard, R. S., Graham-Smith, F., \& Camilo, F. 1996, Nature, 381, 497

Lyne, A. G., Pritchard, R. S., \& Smith, F. G. 1993, MNRAS, 265, 1003

Manchester, R. N., Newton, L. M., \& Durdin, J. M. 1985, Nature, 313, 975

Manchester, R. N., \& Taylor, J. H. 1977, Pulsars (San Francisco: Freeman)

Melatos, A., Peralta, C., \& Wyithe, J. S. B. 2008, ApJ, 672, 1103

Pacini, F. 1967, Nature, 216, 567

Shapiro, S. L., \& Teukolsky, S. A. 1983, Blackholes, White Dwarfs and Neutron Stars: Physics of Compact Objects (New York: John Wiley)

Urama, J. O., Link, B., \& Weisberg, J. M. 2006, MNRAS, 370, L76

Wang, N., Manchester, R. N., Pace, R. T., et al. 2000, MNRAS, 317, 843

Yue, Y. L., Xu, R. X., \& Zhu, W. W. 2007, Adv. Space. Res., 40, 149

Zou, W. Z., Wang, N., Manchester, R. N., et al. 2008, MNRAS, 384, 1063 